\title{
BUIILDING SKINS AS KINETIC PROCESS: SOME PRECEDENT FROM THE FINE ARTS
}

\author{
JULES MOLONEY \\ CRIDA, Faculty of Architecture, Building and Planning \\ The University of Melbourne \\ Carlton, VIC 3010, Australia \\ jmoloney@uninelb.edu.au
}

\begin{abstract}
The aesthetics of form in motion has been a central preoccupation for the visual arts of the twentieth century, culminating in the establishment of kinetic art as a distinct area of activity in the 1950's. Architecture has traditionally resisted 'building kinetics', but has embraced the body in motion, usually on foot negotiating in (subtle) interaction with static form. However, one aspect in which kinetics would appear to be acceptable is at the building periphery, where intelligent facades track sun angles, or moderate air movement in response to internal temperature sensors. On another track is the proliferation of media facades in various guises that transform facades into urban information interfaces or media art works. This paper suggests that both intelligent and media facades set a different agenda for designers who have traditionally worked towards finding the best static mix of performance and elegance. Arguably, intelligent and media facades raises the question of ontology from a designer perspective - what are the design parameters when the outcome is a kinetic system, rather than the traditional static artifact? In order to approach the issue, this paper examines some precedent from kinetic art of the 1960's and contemporary generative arts, for the insight they may provide. These sources then inform a preliminary outline of the range of parameters that may be considered by designers.
\end{abstract}

Keywords: architecture, design, kinetic facades.

\section{Movement at the periphery}

Traditionally, architecture does not move, but it can be designed to embrace the movement of the observer, with Corbusier's promenade architecture an oft cited example. Bois and Shepley's "A Picturesque stroll around Clara-Clara" traces a genealogy of the "peripatetic view", from the Greek revival theories of Leroy, the multiple perspective of Piranesi, Boulee's understanding of the effect of movement, to the Villa Savoye where architecture is best appreciated, according to Le Corbusier " on the move"(56). Such approaches can be captured by the statement: static architecture plus mobile observer results in a kinetic experience. This form of kinetics is referred to here as 'passive', as while there is motion involved, the architecture itself is inert. Passive kinetics also occurs when appearance of surface, form and space is altered by changing environmental conditions. In this case, buildings are designed to accentuate visual transformation in response to different light intensity and direction, the presence of moisture, 
and wind conditions. These two forms of passive kinetics - movement of the observer and transformation due to changes in environmental conditions - are often raised when discussing the merits of actual movement, usually with the innuendo that kinetics is best conceived 'subtly' embedded in static form. While acknowledging the value of passive kinetics to enliven static form, the focus here is on the implications for design when kinetics is 'active' - defined as translation, rotation, and scaling of building components, or the controllable transformation of material properties (for example that enabled by smart materials). The demarcation between this definition of kinetics and the passive approaches outlined above, provides one limit to the discussion. Further limits are the focus on the facade or 'skin', which excludes for example, interactive internal environments such as intelligent rooms, or the idea of re-locatable / reconfigurable buildings championed by the archigram group and others in the 1960's.

Within the above limits, there are currently two areas in which active kinetics are being implemented: intelligent skins are being designed with an environmental science agenda; while in a parallel line of inquiry, there is experimentation with a range of approaches to embodying information, known as media facades. Previously I have argued that there is common ground between environmental facades and data driven skins, in that the design outcome is a process rather then an artefact (683). Rather then being realized as a static object, kinetic skins are manifest as a temporal system, an ongoing process based on: input obtained from sensors or other interface devices; control systems that use logic or emergence; output manifest as moving components or transformation of material properties. The question being explored here, is whether this shift from artifact to kinetic process as an outcome, also suggests an ontological shift. What actually are we designing when we consider the kinetic skin? If we go beyond passive kinetics, based on motion of the observer or optical illusion, and consider the design of active kinetics various questions arise, not the least is it architecture. The view taken, here is that kinetic facades are a development of standard architectural concerns. As evidenced by intelligent facades, the possibilities are for a responsive membrane that adapts to changing environment conditions and user occupancy, continuing the trajectory of functionalism. Media facades by contrast, resurrect the cultural role civic architecture once performed as containers of information. Somewhere between the two, and as yet under theorized are new compositional opportunities for designers, such as that suggested by the poetry of a flock of starlings or a Bill Viola artwork. In summary, the potential of kinetics for building skins are to improve environmental performance, resurrect the cultural role of architecture as embedded information, and allow new forms of composition. However, putting design intent to one side, the proposition here is that regardless of the application, active kinetics raises the question of ontology from a designer perspective - what are the design parameters when the outcome is a process, rather than an object?

In order to approach the question, this paper examines some precedent from kinetic art of the 1960's and contemporary generative arts, for the insight they may provide for the design of kinetic process. Compared to existing precedent in architecture, the range of visual art works that are inherently kinetic is vast. The approach taken is to sample two examples of critique, rather then examine particular works: for the kinetic arts the writing of pioneering artist George Rickey analyses work before the widespread availability of computers; while Melbourne artist / researcher Alan Dorin provides both a useful set of analogy's and a valuable taxonomy of physical process, which he argues underpins the generative electronic arts. These two sources then inform an outline of the range of parameters that may be considered by designers of building skins as kinetic process.

\section{George Rickey's “Morphology Movement"}

Aesthetic outcomes generated by movement can be traced as far back as ancient wind chimes, but the term kinetic art came into being in the twentieth century, with Duchamp's 1920 work 'Rotating Glass Plates' generally acknowledged within western art as the first exhibited work in which the aesthetic is based on physical movement. Other seminal works include Gabo's 'Kinetic Sculpture' (1922) and Moholy Nagy's 'Light Space Modulator' (1930). Anthony Calder dominated the pre-war period with a series of suspended compositions, while the most prolific period for kinetic art was during the 1950's and 1960's. In addition to the continuing popularity of Calder, prominent artists include Schoeffer, Takis, Lye and George Rickey. Rickey trained as a painter in Paris before returning to America in 1949 where he started producing steel sculpture based on a system of meticulously engineered counterweights and bearings, activated by air currents and the pull of gravity. He would continue to refine his work for the next fifty three years, while at the same time teaching and writing in the United States. 
Rickey's essay 'Morphology of Movement: A study of Kinetic Art ', is one of the few attempts at a formal discussion by a leading artist and provides a useful overview of six general directions for the period up until 1963 (222).

(a) Experimentation with optical phenomena, such as moiré effects;

(b) Transformation based on phenomena such as wheel spokes in motion, or through motion of the observer such as effected by polyphonic painting;

(c) Works where the surveyor physically interacts with the work;

(d) Machines where motorized gears and pulleys cause 'orchestrated' movement;

(e) Light play based on coloured light, shadow and reflection;

(f) "'movement 'itself' usually with economy of means and self effacing mechanics".

The first five general directions all seem self evident, but what does Rickey mean by "movement 'itself". The term is a direct quotation from the "Realist Manifesto" in which Gabo observes the limits of Italian Futurism - "It is now obvious to everyone of us that by the simple graphic registration of a row of momentarily arrested movements one cannot re-create movement itself" (221). In his morphology, Rickey argues that the ontology of kinetic art is best addressed by dealing directly with actual movement rather then optical effect. He has a particular dislike of the use of machinery, where repetitive motion generates for him, "a more emphatic stasis" then lack of motion. His argument is that true kinetic works are those where the capacity for motion is designed and is intrinsic, allowing an experience of movement 'itself', without the distractions of mechanics, form, relief, colour or figurative associations (225). Rickey's intuition that kinetics can be clearly distinguished, has been subsequently been proven in medical research, where Zeki and Lamb have determined motion is an autonomous visual attribute, separately processed and therefore one of the visual attributes that have primacy, just like form or colour or depth (607).
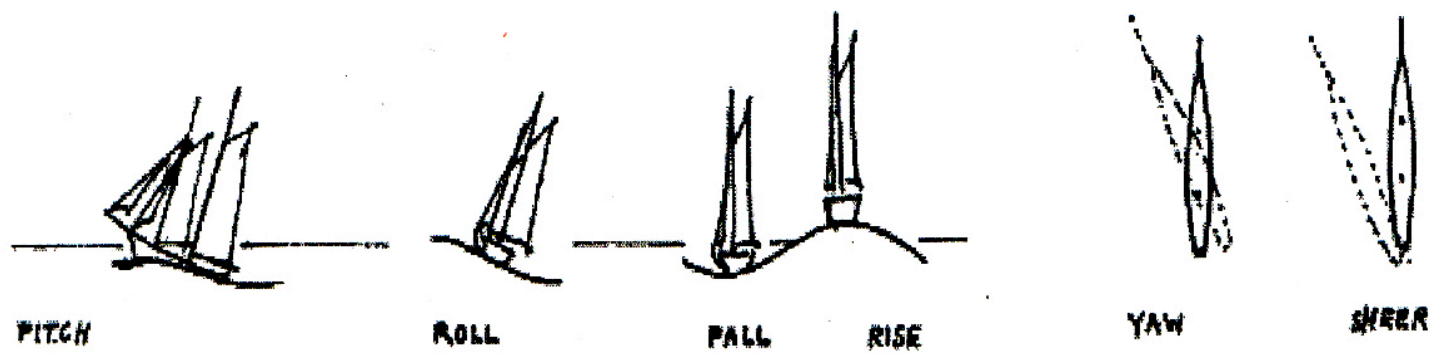

Figure 1: George Rickey - range of movement for a ship at sea.

Rickey's continues his assertion that the essence of kinetic art is the design of movement, by articulating a range of examples: the classic movements of a ship at sea (pitch, roll, fall, rise, yaw, shear) ; vibrating springs; the non periodic movement of a pendulum. For Rickey these are examples of a vocabulary of form in motion, small in number and surprisingly simple, "scarcely more" than the twelve tones of western music (226). Continuing his analogy, this vocabulary is arranged as sequences over time in a similar manner to musical composition. Rickey differentiates kinetic art from music, in terms of its openness to chance "introduced by the movement of the observer, which the artist prepares for but does not predetermine, or by incorporating in the object itself, some factor of fortuitousness" (227). Rickey's example of a ship at sea is simultaneously accurate, in terms of indicating axis of rotation or translations in space, and poetically evokes an aesthetic particular to the circumstance. The ship is an object suspended between fluid and air, with movement by sail or engine dampened by the viscosity of water. The hull is floating, able to simultaneously slide or pivot in any axis, movement is directional, but with the inertia of water and wind mediating the propulsion of screw or sail. There is a particular tempo and the sublime evocation of mass and force resolving in slow motion, an example of "movement itself", that provides a precedent for the articulation of a vocabulary of movement for the design of kinetic skins.

\section{Dorin: painter, playwright, gardener}

A key factor in Rickey's argument for a sophisticated kinetic art was the capacity for a work to accommodate chance, either through making the work open to interaction with environmental 
forces and / or the anticipated but non-predetermined interaction with users. A contemporary art practice that can trace its genealogy back to the idea of indeterminacy as articulated by Rickey, is a form of electronic art described as genetic or evolutionary. For theorist and practising artist Alan Dorin, a key concept that underpins this approach is 'process'.

"A process is any connected sequence of events or actions, it is closely linked to the concept of an algorithm, a sequence of steps for carrying out a task. The concepts of process and algorithm are linked with those of dynamism and change." ("Physicality.." 80)

In a paper that proposes an understanding of physical process is useful for electronic artists, Dorin distinguishes three different types via the example of the painter, the playwright and the gardener: the painter engages with process in terms of colour mixing and application technique but the process is fixed in the final artefact; the production of a play produces a repetitive process, in which the outcome follows a closed script, but is open to nuance in its performance; and the garden in which there is no script but an ongoing process according to natural laws and the 'pruning' of the gardener ("Generative.." 49). These examples, according to Dorin, illustrate the three approaches to using process - fixed, repetitive and ongoing.

Where might we position the architecture in relation to fixed, repetitive and ongoing process? The use of the painter, playwright and gardener to articulate differences between the engagement with process as a means to generate fixed outcomes, and those where process is ongoing, is useful in discussing the traditional role of process in architecture. Process such as sketching and study models are used to design, but once realized the experience of architecture, to varying degrees, is affected by nuances of light, moisture and the position of the observer. We may experience variations based on the two types of passive kinetics outlined in the introduction, but essentially the same script is played out. I would argue that architecture typically has operated between the fixity of painting and the experience of the theatrical script, in which nuances in production may affect experience.

The role of process in the early design stages has been revisited by some contemporary architects, who have embraced digital technology to transform traditional practice. In broad terms there are two approaches being developed - the use of animation techniques where geometric parameters are set in motion and in a second category, those who use generative techniques borrowed from computer science, such as cellular automata or generative algorithms. In the first, the typical approach is to make parametric assemblages where the editing of a part updates the whole. The parameters of this associative geometry are then animated, usually in relation to site constraints or program requirements. The result is architectural form that can be set in motion, as a means to generate a fine grained range of possibilities. In the second approach the outcomes are less predictable, as typically form is generated from the bottom up and evolves according to a set of rules. The process generates a variety, and not unlike the example of the gardener, iterations that meet the program grow and are to varying degrees, pruned by the designer. However outside the design stage this gardening analogy ends - despite sophisticated process being used to generate multiple iterations, only one is realized. The outcome may look like a process, frozen animation or containing genetic references to a family of form, but as architecture it is experienced in typical mode, based on movement of the observer and transformation due to changes in environmental conditions - essentially the same script is played out.

By contrast the kinetic skin may, using Dorin's analogy, be closer to the ongoing process that is the garden. As environmental conditions and user needs change over the course of day, night and seasonal cycles, kinetic skins can potentially undergo significant deformation. Through translation, rotation, and scaling of building components, or the controllable transformation of material properties, the building skin can react and anticipate changes in environmental conditions, or as evidenced by some media facades, the skin can act as a dynamic information interface at an urban scale. There would seem to be the potential for architecture to go beyond the stasis of frozen process or the repetitive script.

\subsection{Dorin's taxonomy of physical process}

Some further insight to the question on what parameters are involved when designing kinetic process, may be gained by considering Dorin's subsequent writing, in which he explores the process of natural systems, which he argues underpins most time based art. He proposes a taxonomy which classifies 'physical' process in a "step towards understanding the relationship between physical processes and time-based art" ("Classification.." 73). He proposes five - 
pulse, stream, increase, decrease, complex. For Dorin kinetics are the outcome of a process, which can be reduced to five actions. Pulse is a "repeating sequence of events" such as the regular pumping of a heart. The spacing between events in a pulse can be of such a scale that it perceived as uniform stream. These occur at the limits of visual perception - a revolving sphere may be rotate so fast that it appears motionless or so slow that process is not apparent. Increase and decrease are relatively self evident forms of process, characterized by forever higher or lower intensity, in which the nature of the change is constant. The final category is complex process, which "forever change into new forms without reiteration". Compared to the regular beat of a pulse, or the smooth change of accelerating or de-accelerating intensity, a complex process "will be different to all future and past states of that system" ("Classification.." 74).

\section{Design parameters for kinetic skins}

The proposition being explored is that the design of kinetic skins sets a different agenda for designers who have traditionally worked towards finding the best static mix of performance and elegance. Rather then a 'finished' architectural surface, the outcome is a kinetic process that interacts with users and in response to changing contexts (environmental and socio-cultural). Arguably, the range of design parameters that need to be considered change the essence of design - the architect is realising a kinetic process as opposed to designing a static object. This shifts the emphasis from 'a' design solution to the specification of parameters: what input and how is this 'sampled' ; the logic of the control system that processes this data; the parameters of the building components or materials that will move in response to the control system. Typically architects engage with the final stage of the kinetic process - the building component or material specification. However if we are to exploit the opportunities offered by kinetic skins designers need to be involved in the design of the system as well as the components. Here the precedent of architects using digital process as a design aid may be useful. Conceptually, the design of kinetic skins is similar to some of these advanced techniques where the designer adjusts parameters to generate a range of outcomes. Except in this case there is no final form, rather the design outcome is kinetic process, from which multiple forms will occur over the life cycle of the building.

From this position, the writing of Rickey and Dorin may be useful for indicating where design decisions occur and the range of parameters that may require consideration. This preliminary outline is intended to identify the general range of factors to be considered, rather then the prescription for any particular design approach. A flaw of all generalist models is that the specificity of each project makes some aspects redundant. However, as a means to articulate the ontological shift that occurs when considering kinetic process as an outcome rather then a design aid, the scope of decisions occur around three interconnected groups of parameters. As the diagram below suggests these are:

(1) Choice of input or sampling;

(2) The manner in which these samples are processed by the control system;

(3) The tectonic, or constructional logic and appearance of the skin.

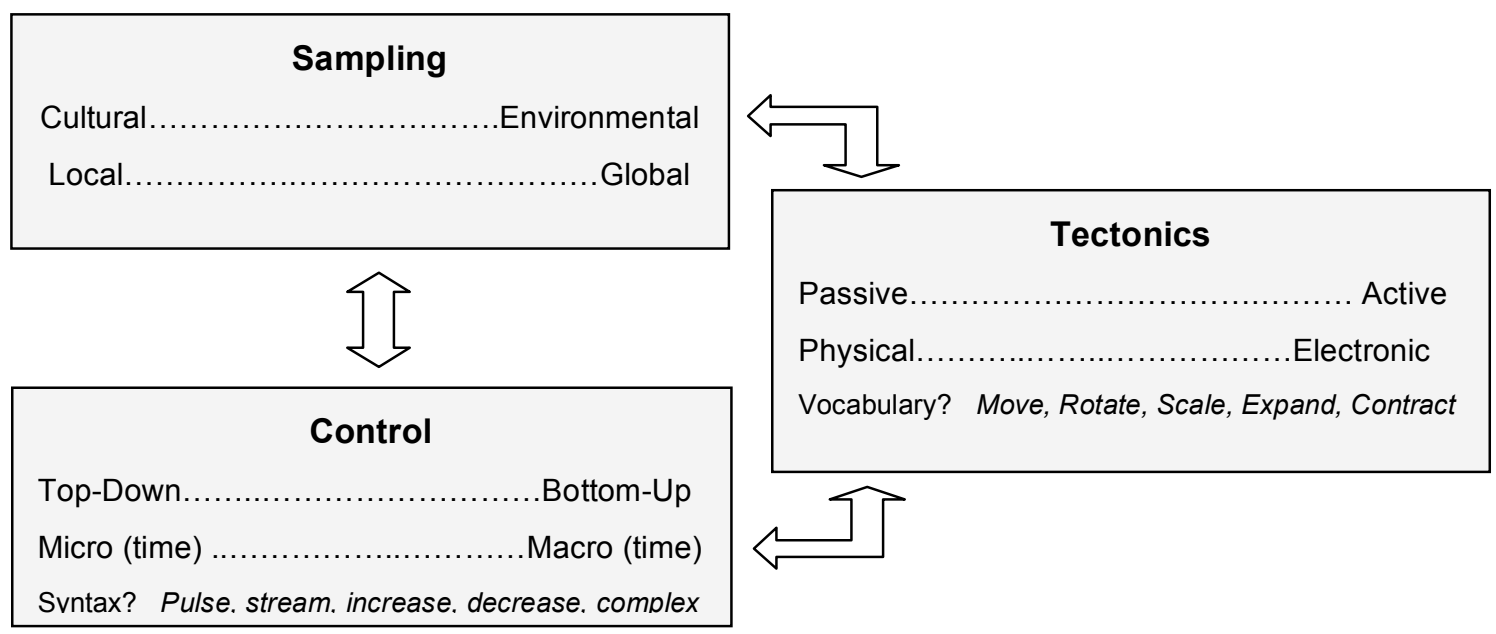

Figure 2: Design parameters for kinetic skins 


\subsection{On sampling}

What data will constitute the physical and what Anders has termed 'virtual space events' of the interactive skin and how will these be captured or sampled? (402) A range of physical sensors are available, tuned to environmental data, physical movement or requiring direct interaction. These can be complimented by data networks that allow access to remote data. Architecture has a long tradition as a form of public art and there exists an opportunity to sample a range of cultural inputs as well as environmental stimuli. Environmental input would necessarily be related to the local, while cultural input could sample both the global and the local. The design of the input mechanism will obviously be dependent on application, but considering this in terms of a full set of possibilities makes explicit that this is a design parameter and specification excludes or includes opportunities.

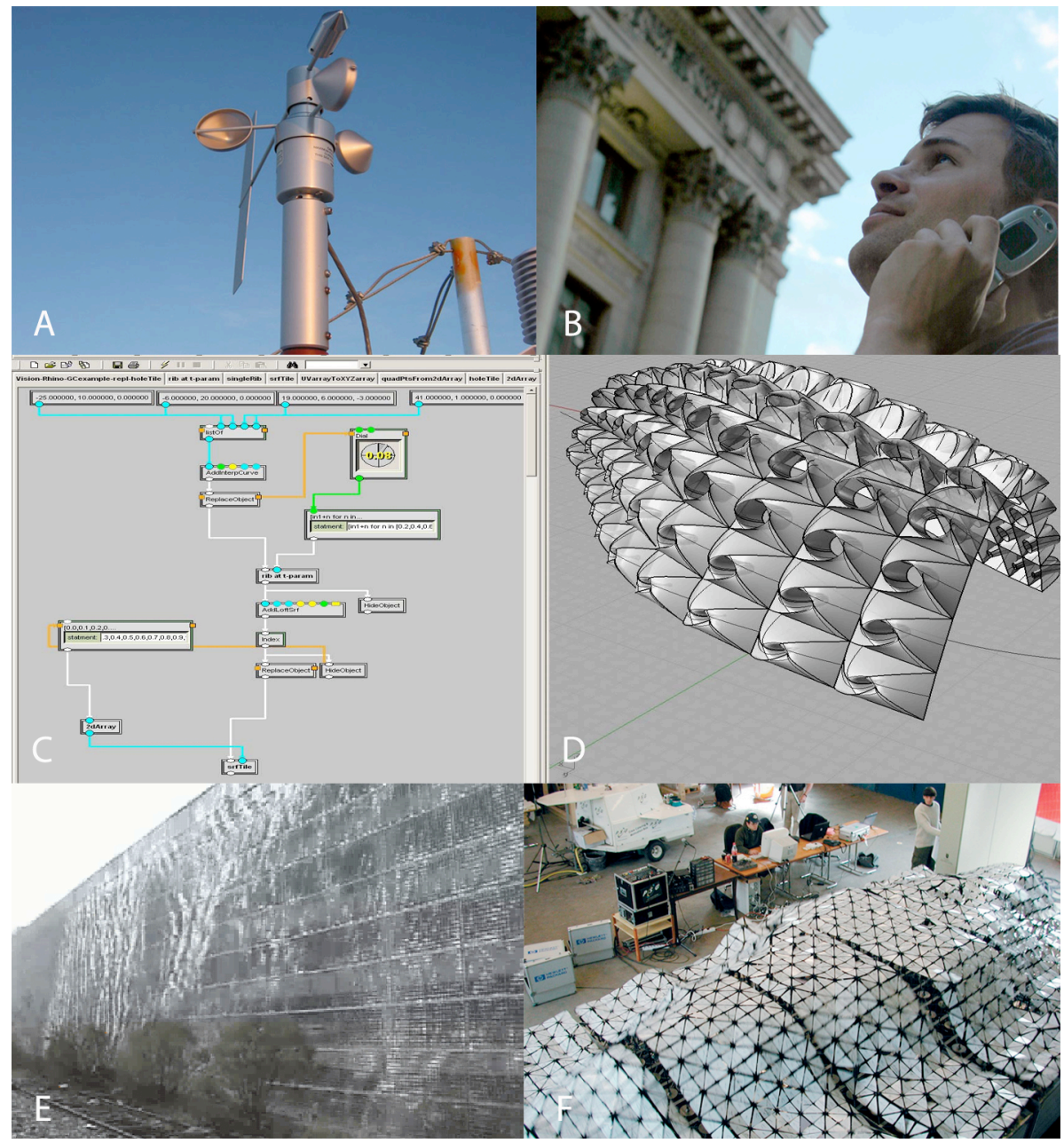

Figure 3: A/B-sampling data from sensors and information portals; C/D-visual programming interface controlling prototype facade (Janssen and Kramer); Etectonic wind wall (Ned Kahn); F-agesis hyposurface (Gaulthorpe et al)

\subsection{On control}

If there is some form of mediation between input and resultant affect, how might this meet aesthetic as well as performative criteria? There may be an opportunity for auto-poesies in which the aesthetic is to a degree, emergent. Alternatively the personal aesthetic of the designer may be embedded in a similar manner to, for example, such proportional systems as used by Palladio or Le Corbusier. Thus the control system would be located within the spectrum of top-down, in which particular criteria are 'directed' and bottom-up approaches where parameters are set for the evolution of behaviour. 
Artists and architects considering the design of interactive skins need to consider foremost that the design of process requires a consideration of performance over multiple time scales. This would range from the micro to the macro in relation to human visual acuity and memory. Input may be directly streamed to output at the micro level of real time response, while simultaneously be processed to create macro scale trends that emerge over a longer period.

Dorin's differentiation between physical and abstract process is useful, given the sampled data could relate to the physical or the abstract and the mediated output could be also be manifest via physical movement or abstract devices such as the media screen. When the control system is related to physical process Alan's taxonomy of pulse / stream / increase, decrease / complex should be considered. This classification could be interpreted as providing a type of formal syntax for the design of process.

\subsection{On tectonics}

What technology is available to implement an interactive skin? Typically, composition in architectural design is based on a tectonic approach in which the aesthetic is largely based on fabrication methods, articulation of joints, and materials. As evidenced by the Arab Institute façade by Jean Nouvel, this attitude to engendering aesthetics can be extended to environmental control systems. Similarly the example of the BIX electronic skin by Peter Cook et al indicates the tectonic design of electronic displays can in itself be important. The interactive skin can be manifest in either physical or electronic form and both require detailed design in terms of their physical appearance as well as their performance. We can make a broad distinction between passive systems with minimal 'mechanics' such as the wind walls of artist Ned Kahn and more complex mechanical systems such as the Agesis Hyposurface.

Finally, if Alan's taxonomy applied to control systems can be seen as the syntax, the range of movement possible with physical systems can, as discussed in relation to Rickey's morphology of kinetic art, be considered a vocabulary of movement. Here the number of discrete actions need to be considered in relation to the chosen tectonic. As suggested by Rickey's example of the ship at sea, the nuance of movement opens up opportunities to develop a particular aesthetic quality. This suggests a subtle range of terms, each tuned to a particular technology, as a conceptual tool to develop and refine kinetic composition.

\section{Post Script}

The above parameters are a preliminary view of a more extended program of research to be undertaken at the University of Melbourne. In order to evaluate and develop this conceptual model for the design of kinetic skins, the next stage will be to undertake a taxonomy of available technology using the 'sampling / control / tectonic' categories. It is anticipated this will produce a useful design resource, but also act as a research methodology, flushing out gaps for the development of new design approaches and technology.

\section{References}

Anders, P. "Cybrid Principles: Guidelines for Merging Physical and Cyber Spaces." International Journal of Architectural Computing 3.3 (2005): pp. 391 - 406.

Bois, P and Shepley J. "A Picturesque Stroll around Clara-Clara." October 29 (1984): pp. 32-36.

Dorin, A. "Classification of Physical Process for Virtual-Kinetic Art." First Iteration. Melbourne, 1999. pp. 68-79.

Dorin, A. "Generative Processes and the Electronic Arts." Organised Sound 6.1 (2001): pp. 4753.

Dorin, A. "Physicality and Notation, Fundamental Aspects of Generative Processes in the Electronic Arts." Proceedings of First Iteration. Ed. Dorin \& McCormack. Melbourne, 1999. pp 80-91.

Moloney, J. "Between Art and Architecture: The Interactive Skin." Information Visualisation '06. London: IEEE Computer Society, 2006. pp. 681 - 86.

Rickey, G.W. "The Morphology of Movement." Arts Journal 22.4 (1963): pp. 220-31.

Zeki, S Lamb, M. "The Neurology of Kinetic Art." Brain 117.3 (1994): pp. 607-36. 